\title{
XVIII. Contributions to the minute anatomy of animals-No. II
}

\section{George Gulliver F.R.S.}

To cite this article: George Gulliver F.R.S. (1842) XVIII. Contributions to the minute anatomy of animals-No. II, Philosophical Magazine Series 3, 21:136, 107-111, DOI: 10.1080/14786444208621494

To link to this article: http://dx.doi.org/10.1080/14786444208621494

册 Published online: 01 Jun 2009.

Submit your article to this journal $\sqsubset \pi$

Џll Article views: 1

Q View related articles $₫$ 
Mr. Gulliver on the Minute Anatomy of Animals. 107

in a hyperbola. But in such cases the arbitrary quantities introduced by integration cannot be satisfied, unless the motion be in confined spaces or narrow canals, such that the coordinates in passing from one point of the fluid to another do not vary independently of each other. These instances are not, therefore, exceptions to the general rule.

Cambridge Observatory, June 15, 1842.

XVIII. Contributions to the Minute Anatomy of Animals. By George Guluiver, F.R.S., \&c. \& c.-No. II.*

\section{On the Nuclei of the Blood-Corpuscles of the Vertebrata.}

BY subjecting the blood of adult mammals to the slow action of a very minute quantity of dilute acetic acid, Dr. Martin Barry states that he has observed nuclei in the corpuscles, which he has depicted in his recent and elaborate researches on the blood (Phil. Trans., 1841, part 2). Yet it seems fair to conclude that there is an essential difference between the blood-corpuscles of mammals and those of the lower vertebrata, since the very same treatment which never fails to show the nuclei in the latter will not exhibit them in the former. This, as I have elsewhere stated (Appendix to Gerber's Anatomy, pp. 13 and 30), does not prove that the corpuscles of mammals include no central matter, although it induced me to believe that these corpuscles have no nucleus like that contained in the corpuscles of the lower vertebrate animals.

When the corpuscles of the oviparous vertebrata are mixed with water, or with dilute or strong acetic acid, the nuclei are instantly exposed in the clearest manner, appearing thick, oval or spherical, and much smaller than their envelopes. Several other vegetable acids, and sulphurous acid, may be used with the same effect; and the nuclei may also be readily shown by gently moistening with the breath some dry blood, which may be again quickly dried so as to preserve the nuclei on the slip of glass for future demonstration. But when the blood-corpuscles of Man and of other mammals, not excepting the oval dises of the Camelidæ (Med.-Chir. Trans, vol.xxiii., and Lancet, vol. ii. p. 101, 1840-41) are treated by any of the means just specified, and precisely under the same circumstances, no similar nuclei will be observed, unless in very young embryos; for the corpuscles of these inclose a temporary and obvious nucleus, which is probably the true analogue of the persistent nucleus of the corpuscles of the oviparous vertebrata.

In the Philosophical Magazine for February 1840, (S.3.

* Communicated by the Author. No. I. will be found in p. 480 of the preceding volume. 
vol. xvi.) p. 106-107, I have noticed that the blood-discs of mammalia become smaller after the removal of their colouring matter by repeated additions of water. Thus some human corpuscles having an average diameter of $\frac{1}{342}$ th of an inch, measured only $\frac{1}{4.800}$ th after the whole of their colouring matter had been separated in this manner, when they appeared flat and pellucid, very faint, and obviously differing in size and general characters from the particles usually described as the nuclei of the blood-corpuscles. No nuclei can be discerned in these washed corpuscles, either by the aid of acids, of corrosive sublimate, or of iodine.

The first part of the preceding observation agrees in some essential points with the results obtained by Sir E. Home (Phil. Trans., 1818, pl. viii. figs. 1, 2, and 3), Schultz (Lancet, 1838-39, vol. ii. p. 713), and Donné (Mandl, Anat. Micros., liv. i. p. 8-9).

If the colouring matter be in like manner washed completely from the blood-corpuscles of the lower vertebrata, both the nuclei and envelopes will remain, the latter becoming quickly circular, and the former also after a few hours. Subsequently the envelopes are scarcely visible, and the colourless matter of the corpuscles, which subsides in the water, appears to be composed chiefly of the nuclei, although with the aid of iodine many of the envelopes may be seen; and these are more or less reduced in size atter a few days, especially in warm weather. Corrosive sublimate affects them very feebly, although it instantly increases the opacity of the washed corpuscles of mammalia. When the former corpuscles have been kept some days in water, the envelopes become very irregular, and hardly perceptible by any means; the size of the nuclei is diminished, and they at length break up into extremely minute molecules.

Dilute muriatic acid renders the nucleus clearly visible in the blood-corpuscles of the oviparous vertebrata. If the corpuscles of a mammal be treated with the same acid, many of them appear shrunk and puckered, notched at the edges, and granulated; some present a distinct central spot, irregular at the margin, like a granular nucleus; others remain smooth at the circumference, often misshapen, and generally with a dark or brilliant central part, according to the focal distance in which they are placed.

The two following figures will illustrate the foregoing observations. The blood-corpuscles of man, and of an adult bird, with some fibrine from the blood of the latter, are represented as magnified about 820 diameters.

Fig. 1. Outlines of blood-corpuscles of Man. In the lower part of the figure, at $A$, corpuscles in pure blood from a prick of the finger: some of them, lying flat, exhibit the central 
spot, which others are without; several are seen on their

Fig. 1.

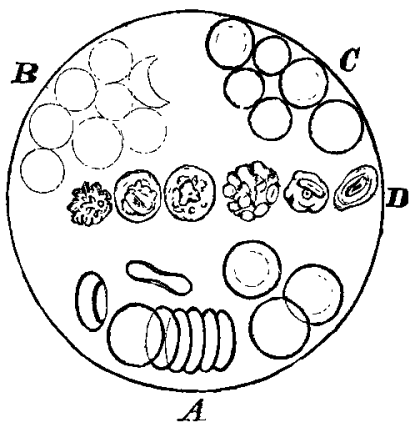

Fig. 2.

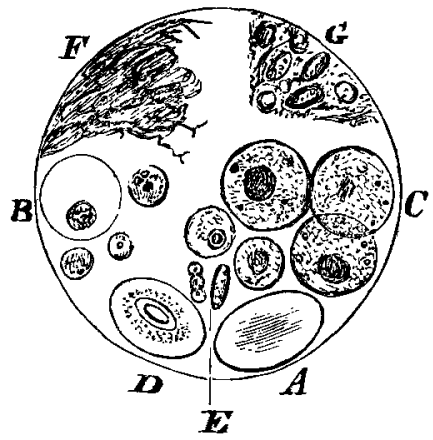

edges collected into a pile; of the two standing separately on their edges, one appears concavo-concave, and the other concavo-convex. B. The corpuscles after thirty hours' washing in cool weather, the water having been changed until the whole of the colouring matter was completely removed. These membranous bases of the discs, are extremely faint; but, as shown at $\mathrm{C}$, they may be rendered very distinct by corrosive sublimate. D. Appearance of fresh corpuscles quickly after treating them with dilute muriatic acid: six of them extend horizontally across the figure.

Fig. 2. Blood-corpuscles and fibrine of a Goose. At A is a fresh unchanged corpuscle. B. Corpuscles after having been washed precisely in the same way as those of the man, but in colder weather; four nuclei are seen, one of which appears to contain minuter granules or nucleoli, and another has a faint envelope. C. The washed corpuscles treated with iodine; some minute molecules adhere to the envelopes, and the nuclei seem to contain nucleoli; the two smaller corpuscles had remained three or four days in the water, at which time many of the envelopes were destroyed, others made irregular in size and shape, and the nuclei reduced to very minute molecules. D. A fresh corpuscle treated with dilute muriatic acid. E. Two oval nuclei obtained by dilute acetic acid from fresh corpuscles, for comparison with the nuclei which appear globular after having been kept in water, as seen at $B$ and $C$. F. Fibrine obtained from fresh blood by washing it in a linen bag. G. The same fibrine, in which a multitude of oval particles, like the nuclei of the blood-discs, are shown by acetic acid.

On the Structure of Fibrine.

In the English version of Gerber's Anatomy, I have de- 
picted organic germs, or objects resembling nucleated nuclei, in clots of fibrine. Those drawings were made from clots which were either pale and opake, or as transparent and colourless as the serum of the blood. I have lately examined the red portions often found towards the edges of such clots, and observed in these coloured parts a multitude of objects like the organic germs above mentioned, but tinged with the colouring inatter of the blood. 'These ruddy bodies appeared to be merely blood-discs entangled in the fibrinous clot and altered in their characters; and hence the pale germs formerly delineated may likewise have been blood-discs still more changed, especially as the corpuscles of the blood are regarded as cells by Schwann, and cell-nuclei by Valentin, while Dr. Barry, as the result of his interesting observations, asks how many tissues are there which the blood-corpuscles may not form?

The corpuscles, of a yellowish or ruddy hue when highly magnified, were contained abundantly in the coloured fibrine: they were rather more irregular in shape than the free corpuscles of the same blood, and differed especially from the latter in exhibiting nuclei when washed either with dilute or strong acetic acid, and even occasionally without the aid of any reagent. The nuclei often appeared as if flattened and with a central point, and sometimes like mere granules; they were commonly grouped together in the centre of the corpuscle, frequently separated, and sometimes scattered about its circumference.

The following figure was made from a minute red part, magnified 800 diameters, of a large, white and very firm clot of fibrine from the heart of a woman, aged 20, who died of puerperal peritonitis and acute pleurisy.

Fig. 3.

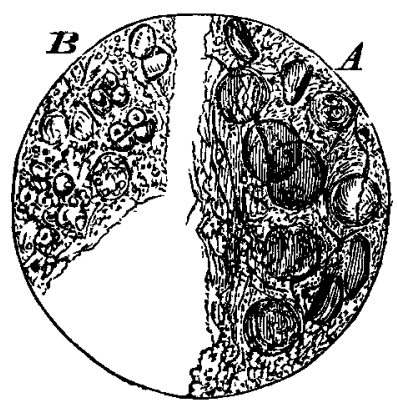

Fig. 3. A. A portion of the coloured fibrinewithout any addition. The corpuscles are contained in a mesh of most delicate fibrils, such as I have formerly described in clots of fibrine 
(Gerber's Anatomy, p. 31); some of the corpuscles, just like misshapen blood-discs, are seen on their edges; others appear mottled, and one exhibits three nuclei. Many minute circular molecules are seen in the fibrine; they were generally from ${ }_{30} \frac{1}{0}, 00$ th to $\overline{T 5,0} \frac{1}{0} \overline{0}$ th of an inch in diameter, but their appearance has not been at all clearly preserved in the engraving. $B$. The same washed with dilute acetic acid; the nuclei of the corpuscles and the minute molecules are distinctly exhibited. Several of the latter are attached to a corpuscle made very faint by the acid.

In fibrine obtained by washing from the blood of the oviparous vertebrata, there is also frequently an appearance of minute fibrils, as shown at F, in fig. 2; but this fibrine is chiefly characterized by its containing numerous particles similar to and probably identical with the nuclei of the blood corpuscles : these particles may often be seen in the fibrine without the addition of any reagent, and acetic acid renders them very plain, as at $\mathrm{G}$ in fig. 2.

XIX. An Account of some Experiments with the Torsionrod, for Determining the Mean Density of the Earth. By Francis Baily, Esq., F.R.S., Vice-President of the Royal Astronomical Society*.

THE author commences his account with a short preliminary history of the subject, and a reference to the previous labours of Maskelyne and Cavendish. He considers the experiments of Maskelyne, on the attraction of the Schehallien mountain, by no means decisive of the question; and with respect to those of Cavendish, by means of the torsion-rod, he is of opinion that Cavendish's object in drawing up his memoir was more for the purpose of exhibiting a specimen of what he considered to be an excellent method of determining this important inquiry, than of deducing a result, at that time, that should lay claim to the full confidence of the scientific world. For, Cavendish himself (who made only 23 experiments), in allusion to this very point, expresses a doubt on the subject, and hints at some further experiments which he had in view, for clearing up some of the irregularities which he had met with. But, as no further account of any subsequent experiments is on record, and as no trace of any new light on this subject can be found amongst Cavendish's papers, the propriety and advantage of repeating the experiments, under

* From the Monthly Notices of the Royal Astronomical Society, having been read May 13 and June 10, 1842. An abstract of Mr. Baily's preliminary paper was given in Phil. Mag. Third Series, vol. xii. p. 233 : a notice of M. Menabrea's paper on Cavendish's Experiments will be found in vol. xix. p. 62. A translation of Laplace's memoir on the mean density of the Earth, in which Cavendish's tesults are examined, was communicated by Dr. Hutton to the First Series, vol. lvi. p. 321.-EDIT. 